\title{
COMMENT
}

\section{Concerning respiration and the carbon-to-nitrogen ratio of a phytoplankton bloom. Comment on Kepkay et al. (1997)}

\author{
Paul Kähler* \\ Institut für Ostseeforschung, Seestraße 15, D-18119 Warnemünde, Germany
}

In the paper 'Respiration and the carbon-to-nitrogen ratio of a phytoplankton bloom' by Kepkay, Jellett \& Niven (1997a), Marine Ecology Progress Series 150: 249-261, the authors present data and interpretations concerning the $\mathrm{C}: \mathrm{N}$ ratios of various organic matter fractions, particulate and dissolved, with the dissolved pool subdivided into a colloidal and a low-molecularweight fraction, and total community respiration during a spring bloom. Their main observation is that total organic carbon and nitrogen dynamics deviate significantly from Redfield stoichiometry (C:N ratios of up to nearly 14). Their main conclusion is that the respiration of colloidal organic matter by bacteria is the prime regulator of this $\mathrm{C}: \mathrm{N}$ ratio. The paper contains a number of omissions, errors, contradictions, and misinterpretations which do not allow this conclusion to be drawn.

The measurement of DON (abbreviations are defined in Appendix 1) in the presence of DIN by hightemperature catalytic oxidation (HTCO) is not trivial. But it is important in the context of the paper. DON was measured like this: 'DON. The high temperature catalytic oxidation instrument of Chen \& Wangersky (1993) was adapted for the colorimetric determination of oxidized nitrogen and used to analyse samples for the total nitrogen content of the dissolved, colloidal and low molecular weight fractions. Typical standard deviations...' (all passages in italics are from Kepkay et al. 1997a). The paper they cited contains the description of the measurement of DOC, not DON. How was DON measured? Why are we not told?! What is the 'oxidized nitrogen content' of the dissolved and other fractions? We are left to guess.

The measurement of DOC by HTCO has in the recent past suffered from analytical difficulties which, when not recognized or ignored, have led to false

•E-mail: paul.kaehler@io-warnemuende.de interpretations of the role of DOC in the sea. Use of this method for DOC has since been subject to rigorous testing and improvement (Sharp 1997). The HTCO measurement of DON has not been consolidated in a comparable way. In a paper making a central point of information obtained by such measurements, one would expect a thorough discussion of this issue or reference to a relevant publication. The authors do not make either of these efforts to convince the reader of the validity of the DON values they report.

The colloidal fraction is given the greatest weight in the discussion and interpretation of the results. But on most sampling days the colloidal fraction is not present in significant concentrations. 'Typical standard deviations' of $2.6 \mu \mathrm{M}$ were associated with the DOC measurements. What, then, is the significance of values obtained by the subtraction of 2 values (DOC minus LOC) each having a 'typical' standard deviation of this size when 7 of 12 of the resulting differences are of the same magnitude as this standard deviation? One wonders how LOC always came out to be lower than DOC (their Table 1). Given this level of precision, a difference between DOC and LOC of $1 \mu \mathrm{mol} \mathrm{l}^{-1}$ (Day 46, first value in their Table 1) has no significance at all. Concerning DON and LON, the situation is similar (their Table 2). For example, at Day 116: 13.4 minus 13.3 is 0.1 , but what is the significance of this number with 'typical' standard deviations of 0.3 of each of the original total dissolved nitrogen measurements? This does not necessarily mean that these measurements are bad, but, even so, there are limits to interpretation set by analytical precision. The authors go a step further (also in error propagation) and calculate the ratio of these numbers, the $\mathrm{C}: \mathrm{N}$ ratio of the colloidal fraction.

But why care about error propagation when the numbers are wrong to start with?! Their Tables 1 \& 2 contain the measured and calculated concentrations of 
$\mathrm{C}$ and $\mathrm{N}$ in the various fractions of the samples from $15 \mathrm{~m}$ water depth. The $\mathrm{C}: \mathrm{N}$ ratios should be obtained by division of the $\mathrm{C}$ and $\mathrm{N}$ concentrations in the respective fractions on any particular day. Doing this for the colloidal fraction (using the values given in Tables 1 \& 2), one arrives at molar $\mathrm{C}: \mathrm{N}$ ratios of between 0.5 and 30 . Kepkay et al. show ratios between 4 and 13 in their Fig. 6C. Also, plotting the C:N ratio of the dissolved fraction from the $\mathrm{C}$ and $\mathrm{N}$ concentrations in Tables 1 \& 2 yields a curve different from that given in their Fig. 6D.

Maybe the C:N ratios suffer from mistakes that crept in at yet an earlier stage, during addition and subtraction when calculating the concentrations of the various fractions of organic matter. In Table 1 the TOC values of Days 74 and 81 are transposed. Other results are given as plots. In Fig. 2, the TOC curve (twice, in $A$ and $B$ ) is obviously not the sum of the DOC (in B) and POC (in A) curves; nor is $\mathrm{CON}$ in Fig. $3 \mathrm{~B}$ the difference between DON and LON at every point (on 2 days LON and DON overlap, but CON, the difference between them, is different from zero). It is difficult to trace what went wrong here and to decide which values can be trusted from a purely arithmetical point of view. The ratios of $\mathrm{C}$ and $\mathrm{N}$ in the different size fractions, especially that of the colloidal fraction, are of importance in the interpretation of the results. The interpretation is certainly not helped by the errors.

Correlations between the $\mathrm{C}: \mathrm{N}$ ratio of the colloidal fraction and total community respiration are reported to be highly significant (their Tables $3 \& 4$ ). The discussion of this finding is a stepwise verbal transformation of a correlation into a causal relationship of prime importance, with no additional information to substantiate this view: p. 258, bottom of right hand column, '...it is important to stress that the correlations cannot be used to quantify the effect of carbon degradation on the bloom's carbon budget.' Next section, 1st paragraph, 'We cannot be sure that only colloid-sized fractions...'; 2nd paragraph, 'We also cannot be sure which organisms...' Summary, 1st paragraph, 'Even though we cannot pinpoint which microbiota or size fractions of organic carbon made the greatest contributions...' The message conveyed in this is obviously: we cannot tell from this who eats what. But after all: 'Microbial respiration is an important regulator... This respiratory regulation of the C:N ratio is focused primarily on the degradation of carbon-rich phytoplankton exudates in the colloidal size fraction... Given the extreme metabolic flexibility of the bacteria, this respiration of colloidal organic carbon (COC)... may....' Bingo! It is bacteria eating colloidal organic carbon. The sequence started with: '. . . we cannot pinpoint which microbiota or size fractions....' As for the COC fraction, its derivation is described above. A reason for reducing total commu- nity respiration via microbial respiration to bacterial respiration is not given (unless 'extreme metabolic flexibility' is accepted, but this may explain anything).

The carbon budget over the bloom period is not balanced. The authors are aware of this, but an explanation is not given. Returning to the last line of p. 258, we read about 'The difficulty of establishing an organic carbon budget for the bloom...' Chlorophyll concentration increases while POC stays low. The change in chl a suggests a POC increase of around $50 \mu \mathrm{M}$ (so does a nitrate consumption of nearly $8 \mu \mathrm{M}$, one might add), but POC increases by only $10 \mu \mathrm{M}$. 'This conflict between low POC concentrations when [sic!] phytoplankton productivity (as defined by chl a [sic!]) was at its height can be resolved by taking sedimentation into account' (on which, by the way, no data are given). Here it is implied that phytoplankton carbon has settled out leaving chlorophyll behind. This is not stated explicitly though, and the conclusion drawn is: 'This leads to an important conclusion: respiration cannot be considered in isolation in its effect on the organic carbon budget. ...it will probably [emphasis added] not be possible to establish a carbon budget' by taking only respiration into account. But, seriously, has anybody ever attempted to build a carbon budget on respiration measurements alone? But this is almost what has been done in the study: respiration has been considered in isolation, not only its effect on the carbon budget, but its effect on the $\mathrm{C}: \mathrm{N}$ ratio, i.e. on the carbon and nitrogen budgets. In this context, recall a passage already cited - '... it is important to stress that the correlations cannot be used to quantify the effect of carbon degradation on the bloom's carbon budget.'this statement is contradicted by the paper itself.

The C:N ratio obviously has a nitrogen component. As for DON, it goes through a minimum during the bloom (their Fig. 3B). This cannot be explained by respiration, and is (consequently?) ignored in the discussion. In attempting to explain why the $\mathrm{N}$ budget is not balanced (about all the DIN used up during blooming is not found in a corresponding rise in TON) selective sedimentation (no data given) is invoked again, along with a somewhat obscure novel pathway: '...nitrogen may have also been removed by rapid recycling...' (p. 257, top of right hand column).

My impression is that the authors have no interest in communicating to the reader a reasonable account of what they have done and what has been going on in Bedford Basin during the bloom. Apart from the observation that the $\mathrm{C}$ and $\mathrm{N}$ dynamics may deviate from Redfield stoichiometry on a small timescale, the paper just conveys a prejudice about the importance of colloids which is not convincingly presented let alone critically questioned by, for example, discussion of alternative interpretations. 
I cannot believe that a phytoplankton bloom is that hard to understand. We are referred to yet another paper by the same authors to appear soon (Kepkay et al. 1997 in press; NB: this paper has since been published and is given in the Literature Cited as Kepkay et al. 1997b). If that one throws light on the carbon and nitrogen budgets, about which many questions are left open, e.g. sedimentation, bacterial numbers, chl a vs phytoplankton carbon, for which information is reported to exist, then this should have been included in the present one. I have the impression, though, that the authors split their study because they wanted to cover as much paper as possible with the least possible amount of data and understanding. This is also a dominant trait of the paper in question here. Most of the plots are shown at least twice (all C:N ratios) and in their Figs. 4, 5 \& 6 there are 2 sets of 5 identical plots of respiration; the plot of chlorophyll concentration at $5 \mathrm{~m}$ is even shown 6 times. This redundancy does not make up for what the paper lacks. It does not make sense.

\section{LITERATURE CITED}

Chen W. Wangersky PJ (1993) A high temperature catalytic oxidation method for the determination of marine dissolved organic carbon and its comparison with the ultraviolet photo-oxidation method. Mar Chem 42:95-106
Appendix 1. Definitions of abbreviations used in text

DIN dissolved inorganic nitrogen

DOM dissolved organic matter

DOC dissolved organic carbon (passing $0.2 \mu \mathrm{m}$ filter)

DON dissolved organic nitrogen (passing $0.2 \mu \mathrm{m}$ filter)

POC particulate organic carbon (retained on GF/F filter)

PON particulate organic nitrogen (retained on GF/F filter)

TOC total organic carbon (sum of particulate and dissolved)

TON total organic nitrogen (sum of particulate and dissolved)

LOC low-molecular-weight organic carbon (passing $10000 \mathrm{kDa}$ nominal pore-size filter)

LON low-molecular-weight organic nitrogen passing $10000 \mathrm{kDa}$ nominal pore-size filter)

COC colloidal organic carbon (difference between dissolved and low-molecular

CON colloidal organic nitrogen (difference between dissolved and low-molecular)

Kepkay PE, Jellett JF, Niven SEH (1997a) Respiration and the carbon-to-nitrogen ratio of a phytoplankton bloom. Mar Ecol Prog Ser 150:249-261

Kepkay PE, Niven SEH, Jellett JF (1997b) Colloidal organic carbon and phytoplankton speciation during a coastal bloom. J Plankton Res 19:369-389

Sharp J (1997) Marine dissolved organic carbon: are the older values correct? Mar Chem 56:265-277 